\title{
Walking Rehabilitation Evaluation Based on Gait Analysis
}

\section{Meiyan Zhang, Jinwei Sun, Qisong Wang, Dan Liu}

School of Instrumentation science and engineering, Harbin Institute of Technology, Harbin, China

Email: zhangmeiyan@163.com, jwsun@hit.edu.cn

How to cite this paper: Zhang, M.Y., Sun, J.W., Wang, Q.S. and Liu, D. (2020) Walking Rehabilitation Evaluation Based on Gait Analysis. Journal of Biosciences and Medicines, 8, 215-223.

https://doi.org/10.4236/jbm.2020.86021

Received: May 18, 2020

Accepted: June 27, 2020

Published: June 30, 2020

\begin{abstract}
With the development of medicine and the improvement of people's living standards, the issue of rehabilitation is getting more and more attention. Gait rehabilitation provides a brand-new treatment method for patients with walking disfunction. It is currently recognized as an advanced rehabilitation medical method in the world. In recent years, the number of patients suffering from dyskinesias in the lower limbs in China has been increasing, and the society's demand for walking rehabilitation treatment is also increasing. The emergence of gait rehabilitation solves the problem of fewer therapists and more patients, reduces the intensity of the therapist's work, and has the incomparable advantage that traditional rehabilitation methods lack. However, because there are no mature related products in China at present, and the prices of foreign products are very expensive, domestic medical institutions have not yet put them into practice. Accelerating the development of gait rehabilitation equipment is of great significance for improving China's medical level, improving the quality of life of patients, and reducing social burden. Usually, high-precision optical sensors are installed on human limbs or using high-speed cameras to capture motion. However, due to the high cost of the equipment, the relative high price of image processing software when processing the collected motion data. In this paper, the acceleration sensors are installed on the human body and the data in a gait cycle can be obtained. After smoothing, using it as the input signal for gait feature extraction and classification. In order to classify normal gait and abnormal gait for evaluation and better walking rehabilitation.
\end{abstract}

\section{Keywords}

Rehabilitation, Gait, Accelerator Sensor, SVM 


\section{Introduction}

As an important kind of biological information, gait is the posture change that people show when walking. It has the characteristics of symmetry, periodicity and individual differences. In many fields such as rehabilitation medicine, bionic design, and human body identification, the research significance and application value of gait have become increasingly prominent. At present, there are two types of research methods for human gait analysis: optical image acquisition technology and sensor information perception technology. Because the latter has the merits of low cost, easy integration, so it is widely used.

Research related to walking rehabilitation issues is of great significance. Its research covers many fields such as rehabilitation medicine, biomechanics, mechanics, mechanical mechanics, electronics, materials science, computer science and robotics, which has become a hot international research field. The meaning of rehabilitation is to restore the normal shape or function of a patient's limb or organ after being traumatized or sick. Rehabilitation engineering is committed to providing patients with auxiliary devices. At present, it has been widely used in rehabilitation nursing, prosthesis and rehabilitation treatment. This not only promotes the development of rehabilitation medicine, but also promotes the development of new technologies and new theories in related fields.

Stroke is one of the main diseases that seriously endanger the health of the elderly. Its main clinical manifestations include hemiplegia, numbness, and tongue speech delay, high disability rate, high recurrence rate, more comorbidities and low cure rate. Studies have shown that about $25 \%$ of survivors in the acute phase after a stroke cannot walk; nearly $50 \%$ of patients require assistance to walk three months after a stroke; the walking speed of patients can only reach about $33 \%$ of healthy peersand step length can only reach about $40 \%$ of healthy peerssix months after a stroke. Abnormal gait will reduce the patient's ability to balance and increase the risk of falling. Gait rehabilitation is of great significance for improving the living standards of stroke patients and reducing the family and socioeconomic burden. At the same time, due to the rapid growth of transportation means, the number of neurological or limb injuries caused by traffic accidents is also increasing. Medical theory and clinical medicine prove that in addition to early surgical treatment and necessary medical treatment, correct and scientific rehabilitation training plays a very important role in the recovery and improvement of limb motor function.

\section{Gait Analysis}

\subsection{Normal Gait}

Normal gait is the posture of the human body when walking. The power of walking mainly comes from the muscles of the lower limbs and trunk. Therefore, walking controlled by the central nervous system also depends on the human body. Through the combination of pelvis, hip, knee, ankle and toes, series of activities are completed. Gait analysis is an examination method to study walking 
laws, aiming to reveal the key links and influencing factors of gait abnormality through biomechanics and kinematics, so as to guide rehabilitation assessment and treatment.

\subsection{Gait Analysis Parameters}

Gait analysis is a special branch of biomechanics. Its parameters can be classified into the following categories: time-distance parameters: including step length, step width, pace, stride, cadence, foot angle, gait cycle, etc.; kinematics parameters: walking mid-hip, knee, ankle Joint motion law (angle change or displacement, speed, acceleration, etc.), pelvic position change law, with joint angle curve, angle-angle diagram, etc.; dynamic parameters: the commonly used is ground reaction force measurement, and the force platform which could measure vertical force component, front and rear force component, and side force component of the ground reaction force; EMG parameters: mainly the electrical activities of the lower limbs muscles during walking; energy parameters: including energy metabolism parameters and mechanical energy parameters. These methods all require certain equipment to detect, such as force platform, EMG, gait analysis system, etc. In clinical practice, gait analysis is widely used in the rehabilitation assessment and treatment of abnormal gait caused by osteoarthropathy and nerve and muscular system diseases, such as physiological lower extremity joint degeneration gait, painful gait caused by arthritis of hip, knee, and ankle, soft tissue injury around the joint or postoperative gait, gluteus maximus gait, mid gluteal muscle gait, design of prosthesis or brace, etc. The combination of biomechanics and kinematics to detect walking can truly reflect gait. Therefore, it is different from other detecting abnormal gaitmethods. The use of human biomechanics and kinematic analysis can make quantitative indicators of abnormal gait. Compared with the traditional rough visual observation method, gait analysis system is currently more advanced, can objectively and quantitatively assess the walking function of the human body, can reflect the patient's rehabilitation function to the greatest extent. The state of rehabilitation function has an important and irreplaceable role in clinical gait analysis.

\subsection{Gait Analysis Method}

Gait analysis is of great guiding significance for lower extremity motor function of stroke patients' rehabilitation methods. According to the results of gait analysis, targeted rehabilitation treatment methods can be implemented for patients, which greatly improves the effectiveness of treatment.

Gait analysis includes the gait cycle and gait phase in time, and the spatial parameters include step length, pace, and pace frequency. There are also biomechanical properties of gait, including joint torque and muscle strength.

Accurate and reliable gait phase detection is critical to gait rehabilitation for patients needing gait impairment. By analyzing the patient's gait phase, the rehabilitation effect of the patient's walking ability can be evaluated after treat- 
ment. Moreover, the study of gait phases can analyze the effect of gait abnormalities in disease analysis and fall prevention. In existing studies, the gait cycle first includes the standing phase and the swing phase. The three sub-phases of the standing phase include the load-bearing period, the middle-bearing stage, and the final stage. The load-bearing phase starts from the Heel Strike, the middle-bearing phase corresponds to the period when the full sole is in contact with the ground, the terminal stance phase starts from the heel starts to leave the ground, and the swing phase is from the toe off the ground to the stage when the heel touches the ground.

In recent years, scientists have studied many methods to divide the gait phase. Among them, the method of motion capture is considered to be the gold standard for gait phase analysis, but it is very expensive and requires high environmental requirements, so it is limited to specific laboratory environments. Researchers have developed a wearable gait analysis system based on different sensors, such as plantar pressure sensors (Force Sensitive Sensors, FSR) [1], joint angle meters, gyroscopes, and accelerometers [2]. In the existing research, the FSR sensor is attached to the heel, toe and sole to measure the plantar pressure during walking. In the gait detection algorithm proposed by this method, support vector machine and linear discriminant analysis method have better performance. Although FSR is inexpensive and easy to use, it is easily broken and inconvenient to wear. Therefore, researchers began to use inertial sensors to collect and analyze gait data. For example, gyroscopes have been used in many studies because their measurement results are not affected by the type. In some other studies, some wearable sensors are also used in combination, such as FSR and gyroscope, accelerometer and gyroscope [3], etc. However, the power consumption of the gyroscope is relatively large, which limits its long-term application in reality. However, accelerometers are relatively low-cost, low-power, and easy to collect data. Therefore, in many studies, accelerometers are used to collect and analyze gait information, and then gait event detection and phase division based on acceleration data.

\section{Experiment Design}

The overall structure of this article is shown in Figure 1.

Signal acquisition is the communication network design and hardware circuit design of the accelerator signal; the data processing is mainly to denoise the collected signal and extract the features; the algorithm analysis is gait recognition based on the analysis of acceleration signals.

\subsection{Hardware System Design}

The main control chip used in this article is Texas Instruments CC3200 of Texas Instruments. This chip is used to control the knee acceleration acquisition chip FlexiForce A301 (Figure 2) for data acquisition. And main features are shown in Table 1. Among them, acquisition chips FlexiForce A301 use GPIO (General 


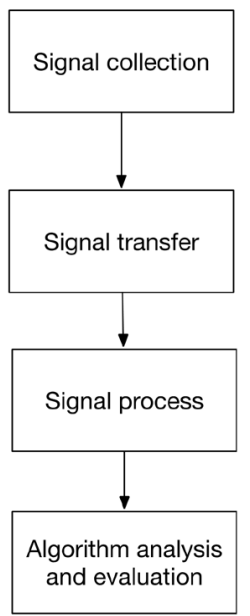

Figure 1. Overall diagram.

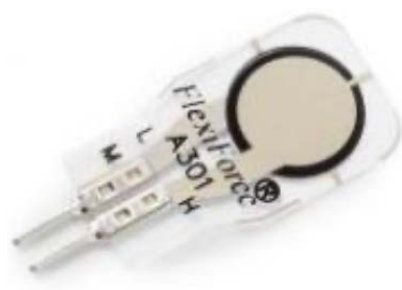

Figure 2. FlexiForce A301.

Table 1. Main features of FlexiForce A301 sensor.

\begin{tabular}{cc}
\hline Parameter & Main features of FlexiForce A301 sensor \\
& Numerical value \\
\hline Length & $25.44 \mathrm{~mm}$ \\
Temperature range & $-40^{\circ} \mathrm{C}-60^{\circ} \mathrm{C}$ \\
Maximum range & $4.4 \mathrm{~N} 111 \mathrm{~N} 445 \mathrm{~N}$ \\
Effective measuring range & $9.53 \mathrm{~mm}($ diameter $)$ \\
Response time & $<5 \mu \mathrm{s}$ \\
Repeatability & $< \pm 2.5 \%$ \\
Lifespan & $>100$ thousand times
\end{tabular}

Purpose Input Output) to communicate with the main control chip CC3200. The wireless Wi-Fi module inside the CC3200 chip can transmit the signals collected by the two sensors.

\subsection{Signal Acquisition}

The operation steps that need to be performed when collecting data information, Figure 3 shows data collection. The collected data can be saved as mat data.

\subsection{Signal Preprocess}

Gait acceleration data cannot be directly used for recognition, it needs to be 


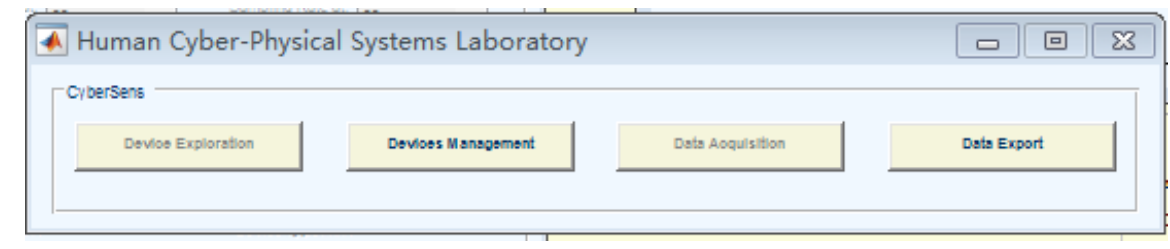

Figure 3. Data collection operation step II.

processed. The process of data preprocessing includes denoising, normalization and division of gait cycle. The current denoising methods mainly include wavelet denoising, low-pass filter and arithmetic average limiting filtering.

1) wavelet transform [4]

It can effectively distinguish the sudden part of the signal and noise. First, the signal is decomposed to remove the interference of the high-frequency part, so the reconstructed signal no longer contains the interference signal, effectively retaining meaningful gait signal characteristics, realizing the noise removal of the non-stationary signal. However, when using the wavelet denoising method, it is necessary to select an appropriate wavelet basis function and decomposition level, otherwise a phase delay will occur.

2) low-pass filter [5]

The currently mainly used is Butterworth low-pass filter. Currently, the third-order and fourth-order are mostly used, and the effect is very good. The signal denoised by Butterworth low-pass filter has a significant phase shift from the original signal. Because the data position in the axis direction of the accelerometer is used to divide the data in the axis direction, no phase shift should exist, is only applicable to the generated phase shift has no effect on the subsequent analysis.

3) arithmetic average limiting

This method uses two consecutive average data to perform the limiting operation. The average value is obtained from multiple acceleration values collected continuously as a group for arithmetic average operation. This filtering method has the advantages of fast operation speed and high smoothness, which prevents the impulsive interference that occurs accidentally and eliminates the interference error.

\subsection{Feature Classification}

Support vector machine (SVM) [6] has strong generalization ability. After training, the complexity of the model is determined by the number of support vectors, not by the dimensions of the data, so fitting problem can be solved well. At the same time, the model trained by SVM completely depends on the support vector. When the points of the non-support vector are removed, the training process is repeated and the same model can still be obtained.

The objective function is:

$$
Q(\alpha)=\sum_{i=1}^{n} \alpha_{i}-\frac{1}{2} \sum_{i, j=11}^{n} \alpha_{i} \alpha_{j} y_{i} y_{j} K\left(x_{i} x_{j}\right)
$$


Classification function:

$$
f(x)=\operatorname{sgn}\left\{\sum_{i=1}^{n} \alpha_{i}^{*} y_{i} K\left(x_{i} x_{j}\right)+b^{*}\right\}
$$

The SVM algorithm can be used for binary classification and multiple classification. The test and training samples of binary classification are different from multiple classification. The test set and training set calculated by the support vector machine are composed of feature vectors, and the two are called the training set and test set feature vectors. The testing process of these two categories needs to construct two categories of label training sets and test set feature vectors. The training set and test set feature vectors of each category are different, so the data set needs to be reconstructed for each experiment. The steps of constructing the feature vectors of the category training and test sets are as follows (Figure 4): First, the data of each scene is evenly divided into training and test set data. Then pre-process the training set and test set data, then perform feature extraction using traditional variance (Figure 5) and feature training with SVM, then use the data set to verify the state of walking rehabilitation.

\section{Conclusions}

It can be considered that the movement of the waist of a healthy person while walking reflects the movement of the person's center of gravity, and the acceleration signal in the $\mathrm{z}$ direction is very regular. In the further processing of each row, the acceleration in the $\mathrm{z}$ direction mainly has two peaks. The first peak is that when the heel is in contact with the ground, the human body will experience a large upward acceleration, about $10 \%$ of each step, reaching the peak, also known as "the moment when the heel touches the ground". After that, it gradually weakened. The second peak will form when the front foot touches the

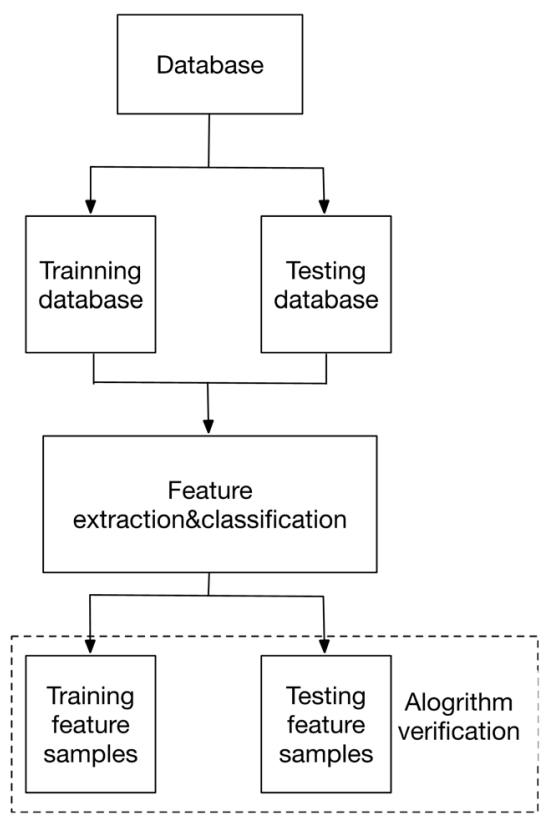

Figure 4. SVM model. 


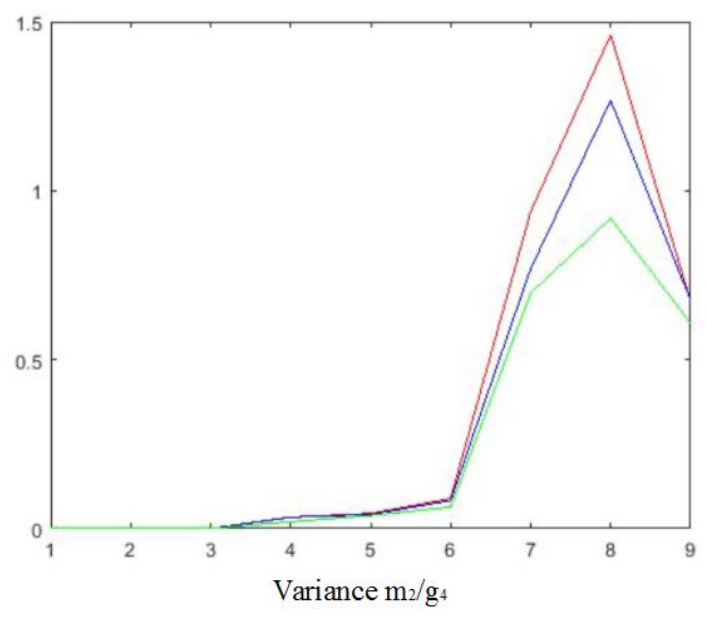

Figure 5. Variance feature extraction.

ground, at which time the human body will accelerate its movement. After that, until the tip of the toe leaves the ground, the acceleration gradually decreases to a negative peak. Before the heel hits the ground, the torso has accelerated forward. After the heel hits the ground, the torso accelerates again until the heel is lifted. After the heel leaves the ground, it gradually accelerates forward, while the body moves forward in parallel. The direction of the lateral acceleration of the torso depends on the limb. A short time after the heel touches the ground, there will be a rapid reverse acceleration, and it will not change direction until midway through the standing phase.

A normal person's thighs to lower legs are fixed with splints to hinder the movement of the knee joint, to imitate patients with walking disability and compare it with normal walking. It can be found that the acceleration signal obviously shows that the amplitude difference between the odd and even steps is large, and the odd and even steps respectively show their periodicity. Therefore, it can be preliminarily determined whether there is an unbalanced movement of the human body in the course of traveling according to this asymmetry of acceleration, and there is a possibility of causing a fall. The more obvious the asymmetry, the weaker the balance ability, the easier it is to cause imbalance, and the easier it is to fall.

Based on the acceleration Based on the law of acceleration signal changes when the human body is walking, it can be concluded that any gait that does not conform to this law is an abnormal gait, which lays a good foundation for walking rehabilitation.

\section{Application}

Gait analysis is an effective means to objectively record the walking style of the human body and systematically evaluate the walking function. Through biomechanics and kinematics could perform rehabilitation assessment and treatment on abnormal gait. Studies have shown that bone and joint disease is one of the main causes of gait disorders, pain and joint relaxation have a significant impact 
on gait. Therefore, it is of clinical value to use gait analysis to assess and treat abnormal gait in patients with knee osteoarthritis from a biomechanical perspective. Fencing athletes' knee joint injuries are thought to be related to the way they move when the sword is made, causing abnormal pressure distribution on the soles of the feet. When the sword is lunged out during the lunge, there is a transition of excessive various and valgus in the foot during the heel cushioning period. The resulting torque may be one of the reasons for the chronic knee injury of fencers. Gait analysis is used to measure the pressure distribution of the fencer's sole when the sword is out of the sword. It provides the basis for clinical rehabilitation and provides a feasible treatment plan for the adjustment of exercise gait.

Gait analysis can also be used to observe changes in gait kinematic parameters and pace. Patients with knee osteoarthritis have knee flexion and extension disorders due to pain and have abnormal gait to reduce pain. Gait analysis provides a basis for clinical abnormal gait rehabilitation assessment and treatment.

\section{Conflicts of Interest}

The authors declare no conflicts of interest regarding the publication of this paper.

\section{References}

[1] Zhang, X.F., Zhao, Y.L. and Xu, Y. (2010) Design and Development of a Novel MEMS Force Sensor for Plantar Pressure Measurement. Third International Conference on Advances in Circuits. IEEE Computer Society.

[2] Gafurov, D., Snekkenes, E. and Bours, P. (2007) Gait Authentication and Identification Using Wearable Accelerometer Sensor. IEEE Workshop on Automatic Identification Advanced Technologies. IEEE. https://doi.org/10.1109/autoid.2007.380623

[3] Mazgut, R., Spanik, P., Koscelnik, J. and Sindler, P. (2014) The Measurement of Balance by the Accelerometer and Gyroscope. Elektro. IEEE. https://doi.org/10.1109/elektro.2014.6847899

[4] Saba, R. and Thurmon, L. (2016) Towards Real-Time Detection of Freezing of Gait Using Wavelet Transform on Wireless Accelerometer Data. Sensors, 16, 475. https://doi.org/10.3390/s16040475

[5] Messier, S.P., Pater, M., Beavers, D.P., et al. (2014) Influences of Alignment and Obesity on Knee Joint Loading in Osteoarthritic Gait. Osteoarthritis and Cartilage. https://doi.org/10.1016/j.joca.2014.05.013

[6] Nakano, T., Nukala, B.T., Zupancic, S., Rodriguez, A. and Nguyen, T.Q. (2016) Gaits classification of Normal vs. Patients by Wireless Gait Sensor and Support Vector Machine (SVM) Classifier. IEEE/ACIS International Conference on Computer \& Information Science. IEEE. https://doi.org/10.1109/icis.2016.7550922 